01

\title{
О связи неэрмитова гамильтониана со стохастическим дифференциальным уравнением в теории открытых систем
}

\author{
(C) A.М. Башаров \\ Национальный исследовательский центр „Курчатовский институт“, \\ 123182 Москва, Россия \\ Кафредра математики и математических методов фризики МФТИ, \\ 141700 Дологопрудный, Россия \\ e-mail: basharov@gmail.com
}

Поступила в редакцию 20.10.2019 г.

В окончательной редакции 20.10.2019 г.

Принята к публикации 01.11.2019 г.

\begin{abstract}
Показано, что ряд задач о распаде частиц, которые описывается неэрмитовым гамильтонианом (соответственно говорят о неунитарной динамике), могут быть корректно и последовательно переформулированы в терминах стохастических дифференциальных уравнений при применении алгебраической теории возмущений. В такой формулировке кинетические уравнения получаются в стандартной схеме теории открытых квантовых систем. Параметры кинетического уравнения совпадают с аналогичными параметрами, описывающими распад частицы при его рассмотрении на основе неэрмитова гамильтониана.
\end{abstract}

Ключевые слова: распад частиц, неэрмитов гамильтониан, кинетическое уравнение, теория возмущений.

DOI: $10.21883 /$ OS.2020.02.48958.284-19

\section{1. Введение}

В последнее время стало модным обсуждать квантовомеханические задачи с точки зрения неэрмитовости гамильтониана, в них возникающего при некоторых описаниях, а также сам неэрмитов гамильтониан и его свойства. Здесь укажем лишь работы [1-3], которые подтолкнули автора к написанию данной работы. Также обсуждают связанные вопросы РТ-симметрии оптических задач с усилением и диссипацией [4,5]. Начиная, наверное, с работ [6,7], неэрмитовы гамильтонианы естественно возникают в описании динамики открытых квантовых систем. До сих пор такие задачи теории открытых систем возникали в основном в теории ядерных реакций, и они решались в основном методом Фешбаха $[8,9]$. В оптике открытые квантовые системы отличаются от типичных ядерных систем тем, что сразу характеризуются использованием в их описании функций и операторов, имеющих разные характерные масштабы временного изменения, но при этом взаимодействие с окружением является малым. Поэтому в отличие от метода Фешбаха в оптике естественным методом исследования оптических открытых систем является метод усреднения КрыловаБоголюбова-Митропольского [10,11], хотя в применении к собственно оптическим системам этот метод оказался весьма громоздким [12]. Для задач взаимодействия атома с классическими оптическими полями разработаны свои методы теории возмущений [13] для вычисления вероятностей многофотонных переходов, отличительной чертой которых является корректное рассмотрение различных и резонансных, и нерезонансных процессов и учет однородного уширения линии. Эти задачи также можно отнести к классу задач с неэрмитовыми гамильтонианами. Однако в недавних работах $[1-3,14]$ ставятся другие вопросы: нахождение собственных функций неэрмитовых гамильтонианов для дальнейшего описания оператора эволюции таких систем.

В данной работе показано, что для определенного класса неэрмитовых гамильтонианов нетрудно записать уравнение Шредингера для оператора эволюции в виде стохастического дифференциального уравнение (СДУ) и решить его, выразив решение через винеровский процесс. Тогда в дальнейшем к задачам с неэрмитовыми гамильтонианами применим мощный аппарат СДУ $[15,16]$. В работе методами алгебраической теории возмущений [17-20] получен эффективный гамильтониан оптической задачи, в которой классическая когерентная электромагнитная волна вызывает переход с дискретного уровня частицы в ее непрерывный спектр, т.е. „распад“ частицы в состояния ее непрерывного спектра. Такая система эффективно описана моделью одноуровневой частицы с динамическим уравнением в виде СДУ (уравнение Шредингера для волнового вектора модели и/или уравнение Шредингера для оператора эволюции). Это СДУ определяет стандартное кинетическое уравнение модели с эрмитовым гамильтонианом, описывающим квантовую эволюцию, и релаксационным оператором в форме Линдблада, описывающим распад исходной системы в непрерывный спектр. При дополнительном пренебрежении обратным приходом с уровней непрерывного спектра на дискретные уровни, кинетические уравнения переходят в уравнения, используемые в теориях с неэрмитовыми гамильтонианами. Таким образом, построено СДУ для оператора эволюции системы и най- 
дено его решение в терминах квантового винеровского случайного процесса

Показано, что перед исследователем, использующим теорию возмущений в поставленной задаче, есть и другой путь - получить в рамках теории возмущений неэрмитов гамильтониан и работать с ним в духе работ $[1-3,14]$. Этот путь можно по форме представить в понятиях алгебраической теории возмущений. Тогда основным отличием подхода, приводящего к неэрмитову гамильтониану, от принципов алгебраической теории возмущений будут правила отбора слагаемых данного порядка теории возмущений. Однако если при этом слагаемые ряда теории возмущений определять согласно принципам алгебраической теории возмущений, то формируется эффективный эрмитов гамильтониан системы, но при этом меняется математический статус динамического уравнения системы - уравнения Шредингера: оно становится корректно определенным как СДУ. Таким образом, продемонстрировано, что подход, в котором возникает неэрмитов гамильтониан, может быть переформулирован в терминах традиционного подхода, когда гамильтонианы должны быть эрмитовыми, но при этом вместо обычных дифференциальных уравнений необходимо работать с СДУ. Некоторое непонимание здесь обычно возникает из-за того, что СДУ использует то же написание дифференциалов, что и обычные дифференциальные уравнения, однако их алгебры дифференциалов отличаются.

Показано, что оба подхода самосогласованы и неэрмитову гамильтониану отвечает корректно определенное СДУ с эрмитовым гамильтонианом. Релаксационные параметры СДУ определяются параметрами неэрмитова гамильтониана. Иначе говоря, стандартно полученное кинетическое уравнение на основе СДУ определяет кинетические уравнения в задачах с неэрмитовым гамильтонианом.

\section{2. Постановка задачи и эффективный гамильтониан}

Пусть имеется квантовая частица с энергетическими уровнями, изображенными на рисунке. Наряду с дискретным уровнем $\left|E_{a}\right\rangle$ имеется ограниченный снизу непрерывный спектр состояний $|v\rangle$, описываемый параметром $v \geq 0$, который отождествляем с энергией состояния непрерывного спектра. Исходный гамильтониан квантовой частицы („атома“, индекс $a$ ) записываем в виде

$$
H_{a}=E_{a}\left|E_{a}\right\rangle\left\langle E_{a}\left|+\int_{0}^{+\infty} \hbar v \rho(v)\right| v\right\rangle\langle v| d v,
$$

где $\rho(v)$ - плотность числа состояний непрерывного спектра частицы.

Оператор $V$ взаимодействия частицы с электромагнитным когерентным полем частоты $\omega$ и напряженности

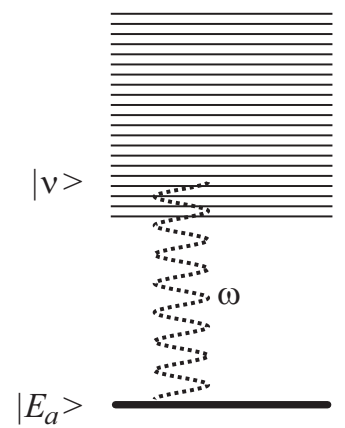

Структура энергетических уровней квантовой частицы и отношение к ним несущей частоты когерентного электромагнитного поля.

электрического поля $E=\mathscr{E}(t) \exp (-i \omega t)+c . c$. примем в электродипольном виде

$$
V=-E d
$$

где

$$
d=\int_{0}^{+\infty} d v d_{v a} \rho(v)|v\rangle\left\langle E_{a}\right|+H . c .
$$

- оператор дипольного момента атома, $\mathscr{E}(t)$ - медленно меняющаяся функция времени по сравнению с быстро меняющейся экспонентой $e^{ \pm i \omega t}$, буквами c.c. и H.c. обозначены слагаемые, комплексно и эрмитово сопряженные предыдущим. Поляризационными и пространственными эффектами, отдачей и переходами в непрерывном спектре квантовой частицы пренебрегаем. Считаем, что рассматриваемые квантовые переходы оптически разрешенные, а матричный элемент оператора дипольного момента в одном и том же состоянии равен нулю (частица не обладает постоянным дипольным моментом). Уровни непрерывного спектра отмечаем их частотой $v$, определяющей энергию как величину $E_{v}=\hbar \nu$.

Считаем, что когерентная волна вызывает переходы в непрерывный спектр, т. е. несущая частота когерентного поля равна частоте перехода с некоторого непрерывного уровня $\left|v_{0}\right\rangle$ на уровень $\left|E_{a}\right\rangle, \hbar \omega=\hbar v_{0}-E_{a}$.

Гамильтониан системы в представлении взаимодействия есть оператор

$$
V(t)=e^{i H_{a} t / \hbar} V e^{-i H_{a} t / \hbar}
$$

взаимодействия квантовой частицы с когерентным полем. Видно, что он содержит слагаемые как быстро, так и медленно меняющиеся во времени:

$$
\begin{aligned}
V(t)= & -\int_{0}^{+\infty} d v d_{v a} \rho(v) e^{i\left(\omega_{v a}-\omega\right) t} \mathscr{E}(t)|v\rangle\left\langle E_{a}\right|+H . c . \\
& -\int_{0}^{+\infty} d v d_{v a} \rho(v) e^{i\left(\omega_{v a}+\omega\right) t} \mathscr{E}^{\mathscr{*}}(t)|v\rangle\left\langle E_{a}\right|+H . c .
\end{aligned}
$$


Это типично для оптической системы, поэтому естественным методом дальнейшего исследования является метод усреднения Боголюбова-Крылова-Митропольского. Представим его в традиционной алгебраической формулировке $[17,19]$ и учтем особенности непрерывного спектра [21].

В качестве динамического уравнения имеем уравнение Шредингера для волнового вектора системы:

$$
i \hbar \frac{d}{d t}|\Psi(t)\rangle=V(t)|\Psi(t)\rangle .
$$

Явное написание аргументов указывает на рассмотрение в представлении взаимодействия.

Перейдем от волнового вектора $|\Psi(t)\rangle$ к новому волновому вектору:

$$
|\tilde{\Psi}(t)\rangle=e^{-i S}|\Psi(t)\rangle
$$

Чтобы форма уравнения Шредингера осталась при преобразовании неизменной, необходимо преобразовать гамильтониан:

$$
\tilde{V}(t)=e^{-i S(t)} V(t) e^{i S(t)}-i \hbar e^{-i S(t)} \frac{d}{d t} e^{i S(t)}
$$

Преобразованный гамильтониан $\tilde{V}(t)$ можно разложить в ряд по генератору преобразования $S(t)$ при помощи формулы Бейкера-Хаусдорфа:

$$
\begin{aligned}
\tilde{V}(t)= & V(t)-i[S(t), V(t)] \\
& -\frac{1}{2}[S(t),[S(t), V(t)]]-\ldots-i \hbar e^{-i S(t)} \frac{d}{d t} e^{i S(t)} .
\end{aligned}
$$

Коммутаторную структуру для оператора дифференцирования по времени мы не стали выписывать, поскольку здесь нужна определенная аккуратность, что отражается в получаемых ниже выражениях.

Оператор дипольного взаимодействия $V(t)$ считаем малым по сравнению с частотой перехода (мала константа взаимодействия). Поэтому представим операторы $S(t)$ и $\tilde{V}(t)$ в виде рядов по константам взаимодействия:

$$
\begin{aligned}
& S(t)=S^{(1)}(t)+S^{(2)}(t)+\ldots, \\
& \tilde{V}(t)=\tilde{V}^{(1)}(t)+\tilde{V}^{(2)}(t)+\ldots
\end{aligned}
$$

Использованы обозначения $S^{(n)}(t)$ и $\tilde{V}^{(n)}(t)$ для слагаемых, имеющие $n$-й порядок по константе взаимодействия когерентного поля.

Нетрудно получить стандартные формулы:

$$
\begin{aligned}
\tilde{V}^{(1)}(t)=V(t)+\hbar \frac{d S^{(1)}(t)}{d t} \\
\tilde{V}^{(2)}(t)=-\frac{i}{2}\left[S^{(1)}(t), V(t)\right] \\
-\frac{i}{2}\left[S^{(1)}(t), \tilde{V}^{(1)}(t)\right]+\hbar \frac{d S^{(2)}(t)}{d t}, \ldots
\end{aligned}
$$

В алгебраической теории возмущений, сформулированной в $[17,19]$, в основу определения слагаемых $S^{(n)}(t)$ и $\tilde{V}^{(n)}(t)$ в (3) положено естественное требование с точки зрения метода усреднения КрыловаБоголюбова-Митропольского - требование отсутствия в $\tilde{V}^{(n)}(t)$ быстро меняющихся во времени слагаемых типа $e^{ \pm i \omega t}$ ит.п. Это приводит к следующему виду оператора $\tilde{V}^{(1)}(t)$ :

$$
\tilde{V}^{(1)}(t)=-\int_{\left(\nu_{0}\right)} d \nu d_{\nu a} e^{i\left(\omega_{\nu a}-\omega\right) t} \mathscr{E}(t)|v\rangle\left\langle E_{a}\right|+H . c .
$$

Здесь под областью интегрирования $\left(v_{0}\right)$ в непрерывном спектре понимается область непрерывного спектра вокруг центральной частоты $v_{0}=\left(E_{a}+\hbar \omega\right) / \hbar$. В результате имеем уравнение для $S^{(1)}(t)$ :

$$
\begin{gathered}
\hbar \frac{d S^{(1)}(t)}{d t}=\int_{0}^{+\infty} d v d_{v a} \rho(v) e^{i\left(\omega_{v a}+\omega\right) t} \mathscr{E} *(t)|v\rangle\left\langle E_{a}\right|+H . c . \\
+\int_{[0, \infty) \backslash\left(v_{0}\right)} d v d_{v a} \rho(v) e^{i\left(\omega_{v a}-\omega\right) t} \mathscr{E}(t)|v\rangle\left\langle E_{a}\right|+H . c .
\end{gathered}
$$

Откуда при допущении адиабатического включения поля получаем

$$
\begin{aligned}
& S^{(1)}(t)=\int_{0}^{+\infty} d v d_{v a} \rho(v) \frac{e^{i\left(\omega_{v a}+\omega\right) t}}{i \hbar\left(\omega_{v a}+\omega\right)} \mathscr{E}^{*}|v\rangle\left\langle E_{a}\right|+H . c . \\
& +\int_{[0, \infty) \backslash\left(v_{0}\right)} d v d_{v a} \rho(v) \rho(v) \frac{e^{i\left(\omega_{v a}-\omega\right) t}}{i \hbar\left(\omega_{v a}-\omega\right)} \mathscr{E}(t)|v\rangle\left\langle E_{a}\right|+H . c .
\end{aligned}
$$

Здесь область интегрирования в непрерывном спектре представляет интервал $[0,+\infty) \backslash\left(v_{0}\right)$, т. е. весь непрерывный спектр за вычетом малой области с центральной частотой $v_{0}$, в которой мы говорим о резонансном взаимодействии когерентной волны с частицей.

Слагаемое второго порядка по взаимодействию $\tilde{V}^{(2)}(t)$ получается в обычном для алгебраической теории возмущений виде

$$
\begin{aligned}
\tilde{V}^{(2)}(t)= & |\mathscr{E}(t)|^{2} \Pi_{a}(\omega)\left|E_{a}\right\rangle\left\langle E_{a}\right| \\
& +|\mathscr{E}(t)|^{2} \int_{0}^{+\infty} d v \Pi_{v}(\omega)|v\rangle\langle v|,
\end{aligned}
$$

с параметрами

$$
\Pi_{a}(\omega)=\int ' d v \rho^{2}(v) \frac{\left|d_{a v}\right|^{2}}{\hbar}\left(\frac{1}{\omega_{a v}+\omega}+\frac{1}{\omega_{a v}-\omega}\right) .
$$

Знак штрих у суммы означает, что в сумме исключены слагаемые с резонансными знаменателями. Например, 
знак штрих у интеграла означает, что исключены области интегрирования, в которых возникают резонансные знаменатели. То есть в интеграле

$$
\int^{\prime} d v \frac{\left|d_{a v}\right|^{2}}{\hbar} \rho^{2}(v)\left(\frac{1}{\omega_{a v}+\omega}\right)
$$

исключена область интегрирования $\left(v_{0}\right)$ вблизи $v_{0}$, в которой знаменатель обращается в бесконечность. Этот интеграл можно понимать в смысле главного значения:

$$
\begin{aligned}
& \int^{\prime} d v \frac{\left|d_{a v}\right|^{2}}{\hbar} \rho^{2}(v)\left(\frac{1}{\omega_{a v}+\omega}\right)= \\
& =-V \cdot p \cdot \int d v \frac{\left|d_{a v}\right|^{2}}{\hbar} \rho^{2}(v)\left(\frac{1}{\omega_{\nu a}-\omega}\right),
\end{aligned}
$$

поскольку матричный элемент $d_{a v}$ дипольного момента слабо меняется как функция $v$ вдали от дна непрерывного спектра, и тогда вблизи $v_{0}$ в области, которая исключена из области интегрирования интеграла со знаком штрих, вклад в интеграл в смысле главного значения равен нулю.

Таким образом, алгебраическая теория возмущений приводит к эффективному гамильтониану системы $H^{E f f}=\tilde{V}^{(1)}(t)+\tilde{V}^{(2)}(t)$. Этот гамильтониан обладает определенной устойчивостью - он состоит из медленно меняющихся во времени компонент и не требует какоголибо усреднения по быстрым переменным. Соответственно эффективный гамильтониан остается неизменным при повторном применении алгебраической теории возмущений.

Подчеркнем, что в первом порядке эффективного гамильтониана присутствует слагаемое, описывающее резонансный переход с дискретного уровня в состояния непрерывного спектра. Его роль рассмотрим в следующем разделе, а ниже обсудим иные требования к получению эффективного гамильтониана системы.

Заметим, что идея эффективного гамильтониана на основе преобразований волнового вектора (1) и исходного гамильтониана (2) не нова и возникла с самого основания квантовой механики [22]. При внимательном рассмотрении подобных идей [17-31] становится понятным, что различные авторы используют разные принципы для формирования слагаемых в рядах (3) и (4). Методу усреднения Крылова-Боголюбова-Митропольского отвечают только подходы алгебраической теории возмущений [15-20]. В первом порядке теорий возмущений разные методы могут давать одинаковые результаты, и этот факт затушевывает различие в подходах, которое проявляется в более высоких порядках теории возмущений.

В основу формирования ряда теории возмущений можно положить требование, чтобы в эффективном гамильтониане системы не было вообще слагаемых, описывающих переходы между дискретными уровнями системы и состояниями ее непрерывного спектра. Обсудим возможные корректные реализации этой идеи в рамках подхода, формулирующего эффективный гамильтониан системы на основе преобразований (1) и (2) исходных волнового вектора и гамильтониана. О таком подходе будем говорить как о теории возмущений для описания распада („распадной“ теории возмущений), чтобы отличать ее от алгебраической теории возмущений.

В теории возмущений для описания распада можно предположить, что в силу „размазанности“ состояния по непрерывному спектру (или иным причинам) константа, отвечающая за переход в непрерывный спектр, представляет собой отдельный параметр малости. Тогда будем считать, что имеют место преобразования, которые также можно записать в форме (1) и (2), но определяемые другим генератором $Q(t)$. То есть в формулах (1) и (2) проводим замены $S(t) \rightarrow Q(t)$, $\tilde{V}(t) \rightarrow \tilde{\tilde{V}}(t), \quad|\tilde{\Psi}(t)\rangle \rightarrow|\tilde{\tilde{\Psi}}(t)\rangle$. При этом представляем операторы $Q(t)$ и $\tilde{\tilde{V}}(t)$ в виде рядов, порядок малости слагаемых которых определяется порядком по взаимодействию дискретного уровня системы с резонансными уровнями из ее непрерывного спектра:

$$
\begin{gathered}
Q(t)=Q^{(1)}(t)+Q^{(2)}(t)+\ldots, \\
\tilde{\tilde{V}}(t)=\tilde{\tilde{V}}^{(0)}(t)+\tilde{\tilde{V}}^{(1)}(t)+\tilde{\tilde{V}}^{(2)}(t)+\ldots
\end{gathered}
$$

Назовем этот параметр малости параметром распада в отличие от параметра малости, определявшего разложение генератора $S(t)$, который будем именовать просто параметром взаимодействия (с когерентным полем).

Разложение генератора преобразования по параметру распада должно начинаться со слагаемого первой степени, иначе не должно быть никаких изменений. Это аналогично алгебраической теории возмущений, формулируемой в представлении Шредингера $[17,19]$. Тогда исходный оператор взаимодействия квантовой частицы разделяем на два слагаемых: $V(t)=V_{a}(t)+V_{d}(t)$. Первое слагаемое $V_{a}(t)$ описывает только дискретные резонансные уровни модели, которые оказались в резонансе с рассматриваемым когерентным полем и/или иными полями, которые могут быть в реальной задаче. Полагаем, что это слагаемое не зависит от параметра распада. Но в рассматриваемой простой модели его нет. Второе слагаемое $V_{d}(t)$ описывает переход в непрерывный спектр, и мы считаем его слагаемым первого порядка по параметру распада. В рассматриваемой простейшей модели

$$
\begin{gathered}
V_{a}(t)=0, \\
V_{d}(t)=-\int_{0}^{+\infty} d v d_{v a} \rho(v) e^{i\left(\omega_{v a}-\omega\right) t} \mathscr{E}(t)|v\rangle\left\langle E_{a}\right|+H . c . \\
-\int_{0}^{+\infty} d v d_{v a} \rho(v) e^{i\left(\omega_{v a}+\omega\right) t} \mathscr{E}^{*}(t)|v\rangle\left\langle E_{a}\right|+H . c . .
\end{gathered}
$$

Тогда

$$
\tilde{\tilde{V}}^{(0)}(t)=0, \quad \tilde{\tilde{V}}^{(1)}(t)=V_{d}(t)+\hbar \frac{d Q^{(1)}(t)}{d t},
$$




$$
\begin{aligned}
\tilde{\tilde{V}}^{(2)}(t)= & -\frac{i}{2}\left[Q^{(1)}(t), V_{d}(t)\right] \\
& -\frac{i}{2}\left[Q^{(1)}(t), \tilde{\tilde{V}}^{(1)}(t)\right]+\hbar \frac{d Q^{(2)}(t)}{d t}, \ldots
\end{aligned}
$$

Требование отсутствия слагаемых, явно описывающих переходы в непрерывный спектр, состоит в выполнении равенства $\tilde{\tilde{V}}^{(1)}(t)=0$. Тогда уравнение

$$
V_{d}(t)+i \hbar \frac{d Q^{(1)}(t)}{d t}=0
$$

является уравнением на определение генератора преобразования $Q^{(1)}(t)$. Затем по выписанным формулам находится оператор $\tilde{\tilde{V}}^{(2)}(t)$, в котором также должны отсутствовать слагаемые, описывающие переход в непрерывный спектр. Эффективный гамильтониан при таком подходе определяется как $\tilde{H}^{E f f}(t)=\tilde{\tilde{V}}^{(0)}(t)+\tilde{\tilde{V}}^{(2)}(t)$. Тогда получаем, что

$$
\begin{aligned}
Q^{(1)}(t)= & \int_{0}^{+\infty} d v d_{v a} \rho(v) \frac{e^{i\left(\omega_{v a}-\omega\right) t}}{i \hbar\left(\omega_{v a}-\omega\right)} \mathscr{E}(t)|v\rangle\left\langle E_{a}\right|+H . c . \\
& +\int_{0}^{+\infty} d v d_{v a} \rho(v) \frac{e^{i\left(\omega_{v a}+\omega\right) t}}{i \hbar\left(\omega_{v a}+\omega\right)} \mathscr{E}^{\mathscr{N}}(t)|v\rangle\left\langle E_{a}\right| .
\end{aligned}
$$

В силу присутствия особенности в первом интеграле в выписанном выражении для генератора $Q^{(1)}(t)$ надо знак H.c. понимать как формальное написание выражения на основе предыдущего, в котором произведены следующие замены: $d_{v a} \rightarrow d_{v a}^{*}=d_{a v}, \mathscr{E}(t) \rightarrow \mathscr{E}^{*}(t),|v\rangle\left\langle E_{a}|\rightarrow| E_{a}\right\rangle\langle v|$ и $i \rightarrow-i$.

Теперь по формуле

$$
\tilde{\tilde{V}}^{(2)}(t)=-\frac{i}{2}\left[Q^{(1)}(t), V_{d}(t)\right]+\hbar \frac{d Q^{(2)}(t)}{d t}
$$

нетрудно найти слагаемое второго порядка по параметру распада. Тогда получаем эффективный гамильтониан в виде

$$
\begin{gathered}
\tilde{H}^{E f f}(t)=|\mathscr{E}(t)|^{2} \tilde{\Pi}_{a}(\omega)\left|E_{a}\right\rangle\left\langle E_{a}\right|, \\
\tilde{\Pi}_{a}(\omega)=\int_{0}^{+\infty} d v \frac{\left|d_{a v}\right|^{2}}{\hbar} \rho^{2}(v)\left(\frac{1}{\omega_{a v}+\omega}+\frac{1}{\omega_{a v}-\omega}\right) .
\end{gathered}
$$

Особенность в подынтегральном выражении учитываем для адиабатического включения поля как

$$
\begin{aligned}
\frac{1}{\omega_{a v}+\omega} & =\frac{-1}{\omega_{\nu a}-\omega} \Rightarrow \frac{-1}{\omega_{\nu a}-\omega-i \delta}= \\
& =V \cdot p \cdot \frac{-1}{\omega_{\nu a}-\omega}-i \pi \delta\left(\omega_{\nu a}-\omega\right),
\end{aligned}
$$

где $\delta=+0$, символ $V . p$. отмечает выражение, интегралы от которого понимаются в смысле главного значения.
Окончательно эффективный гамильтониан приобретает вид

$$
\begin{gathered}
\tilde{H}^{E f f}(t)=\left(|\mathscr{E}(t)|^{2} \Pi_{a}(\omega)-i \Gamma\right)\left|E_{a}\right\rangle\left\langle E_{a}\right|, \\
\Pi_{a}(\omega)=V . p . \int_{0}^{+\infty} d v \frac{\left|d_{a v}\right|^{2}}{\hbar} \rho^{2}(v)\left(\frac{1}{\omega_{a v}+\omega}+\frac{1}{\omega_{a v}-\omega}\right), \\
\Gamma=\pi|\mathscr{E}(t)|^{2} \rho^{2}\left(v_{0}\right) \frac{\left|d_{a v_{0}}\right|^{2}}{\hbar} .
\end{gathered}
$$

Таким образом, эффективный гамильтониан стал неэрмитовым аналогично рассматриваемым в рабо$\operatorname{Tax}[1-3,14]$.

\section{3. Переходы в состояния непрерывного спектра в алгебраической теории возмущений и их описание при помощи стохастического дифференциального уравнения}

Рассмотрим в представлении взаимодействия уравнение Шредингера для оператора эволюции преобразованного вектора состояния с учетом второго порядка алгебраической теории возмущений:

$$
i \hbar \frac{d}{d t} U(t)=H^{E f f}(t) U(t), \quad H^{E f f}(t)=\tilde{V}^{(1)}+\tilde{V}^{(2)}(t),
$$

в котором слагаемые эффективного гамильтониана определены выше формулами (4) и (5).

Удобно представить эффективный гамильтониан в виде суммы других слагаемых, которые описывают только дискретные уровни $H^{(S)}(t)$ (верхний индекс $S$, „система“) и переходы с уровней дискретного спектра на уровни непрерывного спектра $H^{(S-E n v)}(t)$ (верхний индекс $S-E n v$, ,взаимодействие с окружением“ $\left.{ }^{6}\right)$ :

$$
\begin{gathered}
H^{E f f}(t)=H^{(S)}(t)+H^{(S-E n v)}(t), \\
H^{(S)}(t)=|\mathscr{E}(t)|^{2} \Pi_{a}(\omega)\left|E_{a}\right\rangle\left\langle E_{a}\right|, \\
H^{(S-E n v)}(t)=-\int_{\left(v_{0}\right)} d v d_{v a} \rho(v) e^{i\left(\omega_{v a}-\omega\right) t} \mathscr{E}(t)|v\rangle\left\langle E_{a}\right|+H . c .
\end{gathered}
$$

Можно также отдельно рассматривать эффективный гамильтониан окружения:

$$
H^{(E n v)}(t)=|\mathscr{E}(t)|^{2} \int_{0}^{+\infty} d v \Pi_{v}(\omega)|v\rangle\langle v|
$$

Однако если не рассматривать динамику „окружения“ и влияние системы на „окружение“, то он нам не понадобится. Поэтому в дальнейшем ограничимся только эффективным гамильтонианом (14)-(16).

Следует заметить, что, несмотря на проведенное выше разделение рассматриваемой системы на „систему“ и 
„окружение“, параметры эффективного гамильтониана „системы“ все равно зависят от параметров „окружения“ через величину $\Pi_{a}(\omega)(6)$. Аналогичная ситуация имеет место и для обычной двухуровневой модели резонансного взаимодействия квантовой частицы, в которой параметры двухуровневой модели зависят от параметров всех остальных энергетических уровней квантовой частицы $[17,19]$.

Перепишем оператор $H^{(S-E n v)}(t)$, введя операторы рождения $b_{v}^{+}$и уничтожения $b_{v}$ возбуждения энергии $\hbar \nu$ в окружении и „вакуумного“ состояния $|0\rangle$ непрерывного спектра, так что $b_{v}^{+}|0\rangle=|v\rangle$. Считаем, что $\left[b_{v}, b_{v^{\prime}}^{+}\right]=\delta\left(v-v^{\prime}\right)$. Тогда оператор $H^{(S-E n v)}(t)$ записывается в виде

$H^{(S-E n v)}(t)=-\mathscr{E}(t) \int_{\left(v_{0}\right)} d v d_{v a} \rho(v) e^{i\left(\omega_{v a}-\omega\right) t} b_{v}^{+}|0\rangle\left\langle E_{a}\right|+H . c$.

Обратим внимание на формальное сходство операторов $(14)-\left(16^{\prime}\right)$ с операторами задачи о взаимодействии дискретных уровней квантовой системы с широкополосным электромагнитным полем окружения [16,32,33]. Исходя из структуры $H^{(S-E n v)}(t)$ и учитывая аналогичные операторы, описывающие взаимодействие широкополосных полей с квантовыми системами [16,32,33], введем в рассмотрение операторы

$$
\begin{gathered}
b^{+}(t)=\frac{1}{\sqrt{2 \pi}} \int_{-\infty}^{\infty} d v e^{i\left(v-v_{0}\right) t} b_{v}^{+}, \\
B^{+}(t)=\int_{0}^{t} d t^{\prime} b^{+}\left(t^{\prime}\right), \quad d B^{+}(t)=B^{+}(t+d t)-B^{+}(t), \\
b(t)=\frac{1}{\sqrt{2 \pi}} \int_{-\infty}^{\infty} d v e^{-i\left(v-v_{0}\right) t} b_{v}, \\
B(t)=\int_{0}^{t} d t^{\prime} b\left(t^{\prime}\right), \quad d B(t)=B(t+d t)-B(t) .
\end{gathered}
$$

Тогда аналогично квантовым открытым системам, взаимодействующим с широкополосным окружением [16,32], можно говорить о квантовых случайных процессах $B(t)$ и $B^{+}(t)$, дифференциалы Ито $d B(t)$ и $d B^{+}(t)$ которых удовлетворяют обычной алгебре:

$$
\begin{gathered}
d B(t) d B^{+}(t)=d t, \quad d B^{+}(t) d B(t)=0, \\
d B^{+}(t) d t=d t d B(t)=d t d t=0
\end{gathered}
$$

Кроме того, очевидно, что среднее по начальному состоянию „окружения“ - нулевое:

$$
\left\langle d B^{+}(t)\right\rangle=\langle d B(t)\rangle=0 .
$$

Если дополнительно предположить, что матричные элементы дипольного момента $d_{v a}$ и плотность непрерывных состояний $\rho(v)$ слабо меняются вблизи резонансного значения $v_{0}$ (область $\left(v_{0}\right)$ ), то вынося эти значения из-под интеграла и распространяя область $\left(v_{0}\right)$ на всю вещественную прямую, оператор перехода с дискретного уровня в непрерывный спектр можно записать как

$H^{(S-E n v)}(t) d t=-\sqrt{2 \pi} d_{v_{0} a} \rho\left(v_{0}\right) \mathscr{E}(t) d B^{+}(t)|0\rangle\left\langle E_{a}\right|+H . c$.

Тогда уравнение Шредингера (12) становится неопределенным - $B(t)$ и $B^{+}(t)$ являют собой винеровские процессы, которые не дифференцируемы в каждый момент времени [16,32]. Формальное интегральное решение (12)

$$
U(t)=\overleftarrow{T} \exp \left[-\frac{i}{\hbar} \int_{0}^{t}\left(H^{(S)}\left(t^{\prime}\right)+H^{(S-E n v)}\left(t^{\prime}\right)\right) d t^{\prime}\right]
$$

является также неопределенным. Однако если понимать интегралы в смысле Ито как среднеквадратичный предел интегральных сумм с особым выбором значения функции на левых концах интервалов разбиения промежутка интегрирования на $N$ интервалов $\left[t_{i-1}, t_{i}\right]$ :

$$
\int_{0}^{t} \varphi\left(t^{\prime}\right) d B\left(t^{\prime}\right)=m s-\lim _{N \rightarrow \infty} \sum_{i=1}^{N} \varphi\left(t_{i-1}\right)\left(B\left(t_{i}\right)-B\left(t_{i-1}\right)\right),
$$

то для дифференциала Ито $d U(t)=U(t+d t)-U(t)$ из (19) следует соотношение

$$
\begin{aligned}
& d U(t)=\left\{\operatorname { e x p } \left[-\frac{i}{\hbar} H^{(S)}(t) d t+\frac{i \sqrt{2 \pi} d_{\nu_{0} a} \rho\left(v_{0}\right)}{\hbar}\right.\right. \\
& \left.\left.\times\left(\mathscr{E}(t) d B^{+}(t) R_{0 a}+\mathscr{E}^{+}(t) R_{0 a}^{+} d B(t)\right) d t^{\prime}\right]-1\right\} U(t), \\
& R_{0 a}=|0\rangle\left\langle E_{a}\left|, \quad R_{0 a}^{+}=\right| E_{a}\right\rangle\left\langle 0\left|, \quad R_{0 a}^{+} R_{0 a}=\right| E_{a}\right\rangle\left\langle E_{a}\right| .
\end{aligned}
$$

Откуда, раскладывая экспоненту в ряд с учетом алгебры (17), получаем СДУ

$$
\begin{aligned}
d U(t)= & -\frac{i}{\hbar} H^{(S)}(t) U(t) d t \\
& -\frac{\pi}{\hbar^{2}}\left|d_{v_{0} a}\right|^{2} \rho^{2}\left(v_{0}\right)|\mathscr{E}(t)|^{2}\left|E_{a}\right\rangle\left\langle E_{a}\right| U(t) d t \\
& +\frac{i}{\hbar} \sqrt{2 \pi} d_{v_{0} a} \rho\left(v_{0}\right) \mathscr{E}(t)\left(d B^{+}(t) R_{0 a}\right) U(t) \\
& +\frac{i}{\hbar} \sqrt{2 \pi} d_{a v_{0}} \rho\left(v_{0}\right) \mathscr{E}^{*}(t)\left(R_{0 a}^{+} d B(t)\right) U(t) .
\end{aligned}
$$

Уравнение (19) можно формально записать с неэрмитовым гамильтонианом (11):

$$
\begin{aligned}
d U(t)= & -\frac{i}{\hbar}\left(H^{(S)}(t)-i \Gamma\left|E_{a}\right\rangle\left\langle E_{a}\right|\right) U(t) d t \\
& +\frac{i}{\hbar} \Theta d B^{+}(t) R_{0 a} U(t)+\frac{i}{\hbar} \Theta^{*} R_{0 a}^{+} d B(t) U(t),
\end{aligned}
$$




$$
\Gamma=\frac{\pi}{\hbar}\left|d_{v_{0} a}\right|^{2} \rho^{2}\left(v_{0}\right)|\mathscr{E}(t)|^{2}, \quad \Theta=\sqrt{2 \pi} d_{v_{0} a} \rho\left(v_{0}\right) \mathscr{\mathscr { E }}(t) .
$$

При описании этого уравнения важно подчеркнуть следующее.

1. С одной стороны, оператор $H^{(S)}(t)-i \Gamma\left|E_{a}\right\rangle\left\langle E_{a}\right|$ в части взаимодействия с непрерывным спектром совпал с соответствующим слагаемым эффективного гамильтониана „распадной“ теории возмущений.

2. Уравнение (20) описывает унитарную эволюцию всей системы за счет своего особого статуса - уравнение (20) представляет собой СДУ Ито винеровского типа.

Рассмотрим указанные обстоятельства подробнее.

1. Дифференциал Ито от произведения оператора эволюции на его сопряженное $d\left(U(t) U^{+}(t)\right)$ отличается от обычного дифференциала произведения. В силу этой алгебры (17) при взятии дифференциала произведения не выполняется правило Лейбница:

$$
\begin{aligned}
d\left(U(t) U^{+}(t)\right)= & (d U(t)) U^{+}(t) \\
& +U(t) d U^{+}(t)+(d U(t))\left(d U^{+}(t)\right) .
\end{aligned}
$$

В СДУ подразумеваются именно дифференциалы Ито. В вычислениях наряду с $\left(20^{\prime}\right)$ понадобятся дифференциалы Ито оператора:

$$
\begin{aligned}
d U^{+}(t)= & \frac{i}{\hbar} U^{+}(t)\left(H^{(S)}(t)+i \Gamma\left|E_{a}\right\rangle\left\langle E_{a}\right|\right) d t \\
& -\frac{i}{\hbar} \Theta^{*} U^{+}(t) R_{0 a}^{+} d B(t)-\frac{i}{\hbar} \Theta U^{+}(t) d B^{+}(t) R_{0 a} .
\end{aligned}
$$

Тогда с учетом алгебры Ито (17) видно, что

$$
d\left(U(t) U^{+}(t)\right)=0 .
$$

Важным условием выполнения этого равенства, помимо алгебры Ито, служит соотношение

$$
2 \Gamma=|\Theta|^{2},
$$

в котором Г в точности совпало с параметром Г (12) „распадной“ теории возмущения.

2. Статус СДУ позволяет легко и стандартным образом получать кинетическое уравнение для матрицы плотности системы. Преобразования здесь естественны для СДУ, тогда как переход от уравнения Шредингера к уравнению для матрицы плотности в случае неэрмитова гамильтониана содержит подводные камни. Цепочка преобразований с использованием СДУ выглядит так:

$$
\begin{gathered}
d \rho(t) \equiv \rho(r+d t)-\rho(r), \\
\rho(r+d t)=|\Psi(t+d t)\rangle\langle\Psi(t+d t)|= \\
=U(t+d t)|\Psi(0)\rangle\langle\Psi(0)| U^{+}(t+d t), \\
d \rho(t)=d U(t)|\Psi(0)\rangle\langle\Psi(0)| U^{+}(t) \\
+U(t)|\Psi(0)\rangle\left\langle\Psi(0)\left|d U^{+}(t)+d U(t)\right| \Psi(0)\right\rangle\langle\Psi(0)| d U^{+}(t) .
\end{gathered}
$$

Ее результатом является СДУ для матрицы плотности квантовой частицы в представлении взаимодействия с учетом уровней и дискретного, и непрерывного спектров:

$$
\begin{aligned}
& d \rho(t)=\frac{i}{\hbar}\left[\rho(t), H^{(S)}(t)\right] d t-\frac{\Gamma}{\hbar}\left|E_{a}\right\rangle\left\langle E_{a}\right| \rho(t) d t \\
& -\frac{\Gamma}{\hbar} \rho(t)\left|E_{a}\right\rangle\left\langle E_{a}\right| d t+\frac{i}{\hbar} \Theta d B^{+}(t) \rho(t) \\
& -\frac{i}{\hbar} \Theta \rho(t) d B^{+}+\frac{i}{\hbar} \Theta^{*} d B(t) \rho(t)-\frac{i}{\hbar} \Theta^{*} \rho(t) d B(t) \\
& +\frac{1}{\hbar^{3}}|\Theta|^{2} d B^{+}(t) \rho(t) d B(t)+\frac{1}{\hbar^{3}} \Theta^{2} d B^{+}(t) \rho(t) d B^{+}(t) \\
& +\frac{1}{\hbar^{2}} \Theta^{* 2} d B(t) \rho(t) d B(t)+\frac{1}{\hbar^{2}}|\Theta|^{2} d B(t) \rho(t) d B^{+}(t) .
\end{aligned}
$$

Если усреднить уравнение по состояниям только непрерывного спектра, то получим кинетическое уравнение для матрицы плотности частицы (системы) $\rho^{(S)}(t)$, находящейся только на локализованных уровнях:

$$
\begin{aligned}
& \frac{d \rho^{(S)}(t)}{d t}=\frac{i}{\hbar}\left[\rho(t), H^{(S)}(t)\right] \\
& -\frac{\Gamma}{\hbar}\left|E_{a}\right\rangle\left\langle E_{a}\left|\rho^{(S)}(t)-\frac{\Gamma}{\hbar} \rho^{(S)}(t)\right| E_{a}\right\rangle\left\langle E_{a}\right| .
\end{aligned}
$$

При этом мы предположили, что все уровни непрерывного спектра незаселенные, так что обратный переход из непрерывного спектра на дискретные уровни отсутствует. В этом месте можно сказать, что этому также способствуют каскадные переходы с уровней непрерывного спектра на другие уровни непрерывного спектра, возможные процессы столкновений частицы при ее инфинитном движении.

Деление частиц на частицы, заселяющие дискретные уровни, и частицы распавшиеся, заселяющие уровни непрерывного спектра (совершающие инфинитное движение), отличает рассмотренную систему от $[32,33]$. Поэтому уравнение (21), описывающее матрицу плотности атомов на дискретных уровнях, дает уменьшение заселенности дискретных уровней со временем. Это отвечает описанию динамики частиц с неэрмитовым гамильтонианом.

\section{4. Заключение}

Исследования многих задач теории открытых систем и квантовой термодинамики сейчас начинаются с формулировки в качестве исходного уравнения кинетического уравнения в форме Линдблада [34-36]. Как и в представленном анализе, так и в случае общих уравнений Линдблада мы неявно имеем реальный „распадный“ физический процесс (поле), приводящий к появлению неэрмитовой части гамильтониана и/или определяющий линдбладовские операторы релаксации. В случае учета 
в таких задачах воздействия дополнительных полей подход на основе алгебраической теории возмущений и СДУ [37] свидетельствует о появлении слагаемых, представляющих интерференцию учитываемых новых полей с „распадными“. Поэтому требуется определенная аккуратность в использовании исходных уравнений с неэрмитовыми гамильтонианами для анализа воздействия на открытую систему других полей. Роль интерференционных слагаемых здесь может быть как значительной, так и ничтожной. В ряде задач ионизации в классическом поле [13] интерференционными слагаемыми можно пренебречь в силу их малого вклада в вероятности переходов в сильных полях. В других задачах $[34,35]$ с феноменологическим учетом процессов релаксации фазы осцилляторов учет [38] интерференционных слагаемых позволяет избежать парадоксальных результатов [34,35], противоречащих началам термодинамики. А в случае описания процесса затухания моды высокодобротного резонатора учет интерференции поля резонатора и вакуумного поля, определяющего сбой атомной фазы, определяет механизм запирания моды резонатора [39]. Интерференционные слагаемые проявляются также в средах со спектральными особенностями [40]. Представленный нами подход позволяет корректно сопоставлять параметрам феноменологической теории с неэрмитовым гамильтонианом параметры „распадного“ поля, а также строить на основе алгебраической теории возмущений (и определяемом ею СДУ) уравнение для оператора эволюции квантовой системы.

Естественным обобщением предложенной теории служит рассмотрение в еe рамках переноса электрона между квантовыми точками. На основе неэрмитова гамильтониана эта задача рассмотрена в [21]. Результат работы [21] следует при использовании описанного выше подхода на основе СДУ и представленных выше формул. Развитая теория на основе СДУ применима не только к квантовой точке, но и различным их ансамблям, а наличие постоянного дипольного момента и прочих особенностей таких объектов нетрудно здесь учесть.

\section{Финансирование работы}

Работа выполнена при частичной финансовой поддержке Российского фонда фундаментальных исследований (грант № 19-02-00234a).

\section{Конфликт интересов}

Авторы заявляют, что у них нет конфликта интересов.

\section{Список литературы}

[1] Bender C.M. // Contemporary Physics. 2005. V. 46. P. 277.

[2] Longhi S. // J. Phys. A. 2017. V. 50. P. 505201.

[3] Joshi S., Galbraith I. // Phys. Rev. A. 2018. V. 98. P. 042117.

[4] Konotop V.V., Zezyulin D.A. // Opt. Lett. 2014. V. 39. P. 1223.
[5] Konotop V.V., Yang J., Zezyulin D.A. // Rev. Mod. Phys. 2016. V. 88. P. 035002.

[6] Gamow G. // Z. Phys. 1928. V. 51. P. 204.

[7] Siegert A.J. // Phys. Rev. 1939. V. 56. P. 750.

[8] Feshbach H. // Ann. Phys. (NY.). 1958. V. 5. P. 357.

[9] Feshbach H. // Ann. Phys. (NY.). 1962. V. 19. P. 287.

[10] Крылов Н.М., Боголюбов Н.Н. Введение в нелинейную механику. М.: РХД, 2004 (переиздание книги 1937 г.).

[11] Боголюбов Н.Н., Митропольский Ю.А. Асимптотические методы в теории нелинейных колебаний. М.: ГИФМЛ, 1958.

[12] Бутылкин В.С., Каплан А.Е., Хронопуло Ю.Г., Якубович Е.И. Резонансные взаимодействия света с веществом. М.: Наука, 1977.

[13] Делоне Н.Б., Крайнов В.П. Атом в сильном световом поле. М.: Энергоатомиздат, 1984.

[14] Wang H., Lang L.-J., Chong'Y.D. // Phys. Rev. A. 2018. V. 98. P. 012119.

[15] Гардинер К.В. Стохастические методы в естественных науках. М.: Мир, 1986.

[16] Gardiner C.W., Zoller P. Quantum Noise. Berlin: SpringerVerlag, 2004.

[17] Башаров А.М., Маймистов А.И., Маныкин Э.А. // ЖЭТФ. 1983. T. 84. C. 487.

[18] Перлин Е.Ю., Федоров Е.Ю., Кашевник М.Б. // ЖЭТФ. 1983. T. 85. C. 1357.

[19] Maimistov A.I., Basharov A.M. Nonlinear Optical Waves. Dordrecht: Kluwer Academic, 1999.

[20] Bogaevski V.N., Povzner A. Algebraic Methods in Nonlinear Perturbation Theory. Springer, 1991.

[21] Бамаров А.М., Дубовис С.А. // ЖЭТФ. 2005. Т. 128. С. 476.

[22] Van Vleck J.H. // Phys.Rev. 1929. V. 33. P. 467.

[23] Frohlich H. // Phys.Rev. 1950. V. 79. P. 845.

[24] Wentzel G. Quantum Theory of Fields. N.Y.: Interscience Publishers, 1949.

[25] Heitler $W$. The Quantum Theory of Radiation. Oxford: Clarendon Press, 1954.

[26] Бир Г.Л., Пикус Г.Е. Симметрия и деформационные эффекты в полупроводниках. М.: Наука, 1972.

[27] Wagner M. Unitary Transformations in Solid State Physics. Amsterdam: North-Holland, 1986.

[28] Башаров А.М. Фотоника. Метод унитарного преобразования в нелинейной оптике. М.: МИФИ, 1990.

[29] Wegner F. // Ann.Phys. 1994. V. 3. P. 77.

[30] Glazek S.D., Wilson K.G. // Phys. Rev. D. 1993. V. 48. P. 5863.

[31] Glazek S.D., Wilson K.G. // Phys. Rev. D. 1994. V. 49. P. 4214.

[32] Gardiner C.W., Collet M.J. // Phys. Rev. A. 1985. V. 31. P. 3761.

[33] Gardiner C.W. // Phys. Rev. Lett. 1986. V. 56. P. 1917.

[34] Werlang T. et al. // Phys. Rev. A. 2008. V. 78. P. 053805.

[35] Dodonov A.V. // Phys. Scr. 2012. V. 86. P. 025405.

[36] Levy A., Kosloff R. // EPL. 2014. V. 107. P. 20004.

[37] Бамаров А.М. // ЖЭТФ. 2012. Т. 142. С. 419.

[38] Basharov A.M. // J. Phys. CS. 2015. V. 613. P. 012007.

[39] Basharov A.M. // Phys. Lett. A. 2012. V. 376. P. 1881.

[40] Башаров А.М. // Письма в ЖЭТФ. 1999. Т. 70. С. 434. 